\title{
A New Interface Method for Hyperbolic Problems with Discontinuous Coefficients. One-Dimensional Acoustic Example.
}

\author{
$\underline{\text { Jö̈L PIRAUX - BRUnO LOMBARD }}$ \\ Laboratoire de Mécanique et d'Acoustique \\ 31, chemin Joseph Aiguier \\ 13402 MARSEILLE CEDEX 20 - FRANCE
}

\begin{abstract}
A new numerical method, called the Explicit Simplified Interface Method (ESIM), is developed in the context of acoustic wave propagation in heterogeneous media. Equations of acoustics are written as a first-order linear hyperbolic system. Away from interfaces, a standard scheme (Lax-Wendroff, TVD, WENO...) is used in a classical way. Near interfaces, the same scheme is used, but it is applied on a set of modified values deduced from numerical values and from jump conditions at interfaces. It amounts to modify the scheme so that its order of accuracy is maintained at irregular points, despite the non-smoothness of the solution. This easy to implement interface method requires few additional computational resources and it can be applied to other partial differential equations.
\end{abstract}

Keywords: Acoustics; heterogeneous media; Lax-Wendroff, TVD and WENO schemes; Interface Methods; discontinuous coefficients.

AMS Classification: 65M06, 35L05

\section{Introduction}

The propagation of acoustic waves in a one space dimension heterogeneous perfect fluid medium is considered. The density and the sound velocity are piecewise constant. The acoustic velocity $u$ and the acoustic pressure $p$ are computed on a uniform grid, even if interfaces do not coincide with grid points. The goal of this presentation is to develop an accurate method for the computation of $u$ and $p$ near interfaces, where they are not smooth.

Even if the one-dimensional problem is academic, it has some interesting applications. It is often used as a simple model in seismology or ocean acoustics, in which the ocean bottom is described as a multilayered fluid medium [1]. Discontinuities between sediment layers can be considered to depend only on the depth below the sea floor. Furthermore, the description of a multilayered medium possibly involving hundreds of interfaces is useful for modelling sound propagation in a fluid medium with many inclusions or bubbles.

Equations of acoustics are usually written as a second-order scalar wave-equation for $u$ or $p$. It is however useful to compute $u$ and $p$ simultaneously by considering the first-order hyperbolic system:

$$
U_{t}+A(x) U_{x}=0
$$

where components of $U(x, t)$ are $u$ and $p$, and $A(x)$ is a matrix involving physical parameters.

Many schemes can be used for the resolution of (1). A general introduction can be found in LeVeque's book [10]. Three schemes of increasing precision are used in this paper: Lax-Wendroff, TVD and WENO. They use values of the density and of the sound speed on discrete points or averaged values of these coefficients on grid cells: as a consequence, they 
do not describe accurately the position and the shape (in two or three space dimensions) of interfaces cutting grid cells. Furthermore, $u$ and $p$ are not smooth across interfaces: it results in a loss of precision, increasing with the number of interfaces and with contrasts.

It is then interesting to use an interface method, like the Immersed Interface Method (IIM) [2, 12, 16], or the Explicit Jump Immersed Interface Method (EJIIM) [15]. These numerical methods ensure a given accuracy at grid points near interfaces but they are difficult to implement with high order schemes, and they introduce some numerical artefacts in various cases.

A new approach called the Explicit Simplified Interface Method (ESIM) is proposed. The same scheme is used everywhere, but some values involved in time-marching near interfaces are modified. These modified values are deduced from numerical values of $U$ at grid points near the interface and from jump conditions, so that the loss of accuracy due to the presence of interfaces is avoided.

The main goal of this one-dimensional study is to illustrate clearly ideas underlying the ESIM. Similar ideas can be used for other partial differential equations, such as electromagnetic or fluid mechanic ones. The paper is divided as follows. In Section 2, conservation laws, jump conditions and some numerical schemes are shortly recalled. In the same Section, advantages and drawbacks of interface methods like the IIM and the EJIIM are developed. The ESIM is presented in Section 3. The Section 4 consists in numerical experiments including too a $2 \mathrm{D}$ example. The Section 5 concludes the paper by recalling the three key-stages of the method.

\section{Numerical schemes and interface methods}

\subsection{One-dimensional acoustic equations}

\section{Conservation laws:}

The linearization of mechanic equations in an ideal fluid leads to:

$$
\begin{aligned}
& \rho u_{t}+p_{x}=0 \\
& p_{t}+\rho c^{2} u_{x}=0,
\end{aligned}
$$

where $u(x, t)$ is the acoustic velocity and $p(x, t)$ is the acoustic pressure. The density is $\rho(x)$, the sound speed is $c(x)$. Setting:

$$
U(x, t)=\left(\begin{array}{c}
u \\
p
\end{array}\right), \quad A(x)=\left(\begin{array}{cc}
0 & \frac{1}{\rho} \\
\rho c^{2} & 0
\end{array}\right),
$$

a first-order linear hyperbolic system is obtained:

$$
U_{t}+A(x) U_{x}=0
$$

\section{Jump conditions:}

The location of abrupt changes in $\rho$ and/or $c$ is called an interface. Unlike gas dynamics problems, interfaces do not move. In one, two and three space dimensions, an interface is respectively a point, a curve and a surface. This definition can be extended to boundaryvalue problems or singular sources [12], [15]. To keep it simple, only one interface at $x=\alpha$ is considered in Section 2 and Section 3. Physical parameters are piecewise constant:

$$
(\rho, c)=\left\{\begin{array}{lll}
\left(\rho^{-}, c^{-}\right) & \text {if } & x \leq \alpha \\
\left(\rho^{+}, c^{+}\right) & \text {if } & x>\alpha .
\end{array}\right.
$$


It leads to constant matrices $A^{-}$if $x \leq \alpha$ and $A^{+}$otherwise:

$$
A^{-}=\left(\begin{array}{cc}
0 & \frac{1}{\rho^{-}} \\
\rho^{-}\left(c^{-}\right)^{2} & 0
\end{array}\right), \quad A^{+}=\left(\begin{array}{cc}
0 & \frac{1}{\rho^{+}} \\
\rho^{+}\left(c^{+}\right)^{2} & 0
\end{array}\right) .
$$

Writing for any function $f(x, t)$ :

$$
[f]=\lim _{x \rightarrow \alpha^{+}} f(x, t)-\lim _{x \rightarrow \alpha^{-}} f(x, t)
$$

acoustic jump conditions are: $[u]=0,[p]=0$. It follows:

$$
[U]=0 .
$$

Jump conditions of spatial derivatives of $U$ are deduced from (4) and (8). By induction, we can easily verify that, on both sides of $\alpha$, we get for all $k \geq 0$ :

$$
\begin{aligned}
& \frac{\partial^{2 k}}{\partial t^{2 k}} U=c^{2 k} \frac{\partial^{2 k}}{\partial x^{2 k}} U \\
& \frac{\partial^{2 k+1}}{\partial t^{2 k+1}} U=-c^{2 k} A \frac{\partial^{2 k+1}}{\partial x^{2 k+1}} U .
\end{aligned}
$$

The relation (8) is true for all $t$. Differentiating (8) with respect to $t$, exchanging the order of spatial jumps and time derivatives, and using (9), lead to jump condition of any order. Setting:

$$
D_{2 k}=\left(\frac{c^{-}}{c^{+}}\right)^{2 k} I_{2}, \quad D_{2 k+1}=\operatorname{diag}\left(\frac{\rho^{-}}{\rho^{+}}\left(\frac{c^{-}}{c^{+}}\right)^{2 k+2}, \frac{\rho^{+}}{\rho^{-}}\left(\frac{c^{-}}{c^{+}}\right)^{2 k}\right),
$$

where $I_{2}$ is the 2 -by-2 matrix identity, jump conditions are for all $k \geq 0$ :

$$
\begin{aligned}
& \lim _{x \rightarrow \alpha^{+}} \frac{\partial^{2 k}}{\partial x^{2 k}} U(x, t)=D_{2 k} \lim _{x \rightarrow \alpha^{-}} \frac{\partial^{2 k}}{\partial x^{2 k}} U(x, t), \\
& \lim _{x \rightarrow \alpha^{+}} \frac{\partial^{2 k+1}}{\partial x^{2 k+1}} U(x, t)=D_{2 k+1} \lim _{x \rightarrow \alpha^{-}} \frac{\partial^{2 k+1}}{\partial x^{2 k+1}} U(x, t) .
\end{aligned}
$$

\subsection{Numerical schemes}

Given a uniform grid with time step $\Delta t$ and spatial mesh size $\Delta x$, we look to an approximation $U_{j}^{n}$ of $U\left(x_{j}, t_{n}\right)$ at the point $\left(x_{j}=j \Delta x, t_{n}=n \Delta t\right)$, called afterwards numerical value. Two-level explicit finite-difference schemes are used here. They are $(2 k+1)$-point spatially centered schemes, where $k$ is called the width of the scheme, and time-marching is performed in two or more stages. They follow the CFL condition of stability:

$$
\mathrm{CFL}=\max c\left(\frac{\Delta t}{\Delta x}\right) \leq 1
$$

To keep it simple, a given scheme is referred to as $\mathcal{S}$. Time-marching of a two-stage scheme $\mathcal{S}$ is written abstractly at $x_{j}$ :

$$
U_{j}^{n+1}=U_{j}^{n}+\mathcal{H}\left(U_{j-k}^{n}, \ldots, U_{j+k}^{n}\right),
$$

where the discrete operator $\mathcal{H}: R^{2 \times(2 k+1)} \rightarrow R^{2}$ is continuous [7]. Among two-stages schemes, we distinguish the 3-point Lax-Wendroff scheme $(k=1)$ and 5 -point TVD schemes $(k=2)$. The Lax-Wendroff scheme is easy to implement and requires few computational resources but it suffers from numerical dispersion (see Figure 3-b). High order TVD schemes, based on the use of nonlinear flux limiters, allow to reduce the spurious oscillations [10] but 
flatten the crests of waves (see Figure 3-c). The MC-limiter is used in Section 4 [9]. Both schemes are second-order accurate in smooth regions.

A better quality is obtained with multi-stage schemes as Essentialy Non Oscillatory (ENO) schemes and WENO (Weighted ENO) schemes [4, 10]. They are based on distinct discretizations of spatial and time derivatives of (4). We have implemented a spatial interpolation on three points, called WENO $5(k=3)$, which is fifth-order accurate in smooth regions [8]. It involves some regularity parameters $\epsilon=10^{-6}$ and $p=2$ ( $p$ has obviously nothing to do with the pressure). The time discretization is implemented by a fourth-order Runge-Kutta method.

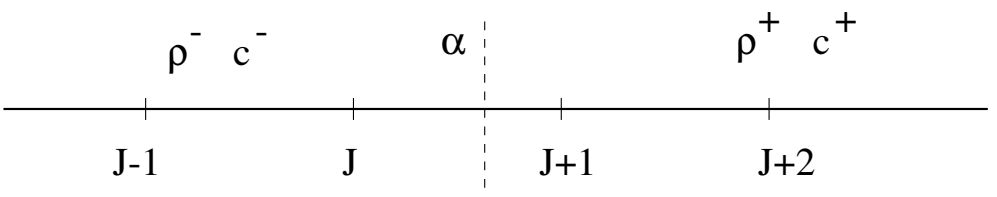

Figure 1: Interface 1-D

The interface lies between two grid points: $x_{J} \leq \alpha<x_{J+1}$, as shown in Figure 1 . A point $x_{i}$ is called irregular if time-marching at $x_{i}$ uses one or more numerical value on the other side of the interface. Otherwise, a point is called regular. For spatially centered $(2 k+1)$-point schemes used here, the set of irregular points is $\left\{x_{J-k+1}, \ldots, x_{J+k}\right\}$.

\subsection{Interface methods}

\section{Presentation:}

In one space dimension, $u$ and $p$ are in $\mathcal{C}^{0}$ across interfaces (11). In two space dimensions, the acoustic velocity component tangential to the interface is discontinuous across the interface; the acoustic pressure and the normal acoustic velocity component are in $\mathcal{C}^{0}$. Thus the numerical resolution of (4) with interfaces requires schemes specially designed for the computation of non-smooth solutions. Schemes based on naive finite-difference approximations may give poor results. For example, the Lax-Wendroff scheme results from replacing spatial derivatives $U_{x}$ and $U_{x x}$ by centered finite-difference evaluations. This is valid only if $U(x, t)$ is smooth in the interval $[x-\Delta x, x+\Delta x]$. This is not the case at irregular points $x_{J}$ and $x_{J+1}$, leading to a loss of accuracy.

Even with schemes especially designed for the computation of discontinuous solutions, as TVD or WENO schemes [5], [6], [11], the numerical solution suffers from a loss of accuracy when interfaces cut through grid cells [16]. This loss of accuracy is due to two reasons. Firstly, schemes do not describe accurately the position (and the shape, in two or three spaces dimensions) of interfaces. Secondly, the spatial order of accuracy is always reduced at irregular points, because of the non-smoothness of the solution.

Moreover, schemes do not take into account jump conditions at interfaces. Therefore, the study of original jump conditions problems (like the propagation of elastic waves across imperfect bonded media [14], or advection problems like those defined in chapter 2 of [16]) is difficult. Lastly, numerical instabilities can appear for high contrasts in material properties.

Schemes must be modified at irregular points in order to eliminate those problems. The resulting scheme is called an interface method, because it is based on jump conditions of $U$ at the interface. Two interface methods are now shortly recalled.

\section{The Immersed Interface Method (IIM):}

One way is to write explicitly a new scheme at irregular points: it is the aim of the Immersed Interface Method (IIM). It was developed by Li and LeVeque [12], extended to 
acoustics and elasticity by Zhang [16], [17], and to Navier-Stokes by Calhoun [2]. In [16], the Lax-Wendroff scheme is used at regular points. A new 3-point finite-difference scheme is written at irregular points:

$$
\begin{aligned}
& U_{J}^{n+1}=U_{J}^{n}+\frac{\Delta t}{\Delta x}\left(\Gamma_{J, 1} U_{J-1}^{n}+\Gamma_{J, 2} U_{J}^{n}+\Gamma_{J, 3} U_{J+1}^{n}\right) \\
& U_{J+1}^{n}=U_{J+1}^{n}+\frac{\Delta t}{\Delta x}\left(\Gamma_{J+1,1} U_{J+2}^{n}+\Gamma_{J+1,2} U_{J+1}^{n}+\Gamma_{J+1,3} U_{J}^{n}\right) .
\end{aligned}
$$

$\Gamma$ 's are 2-by-2 matrices, defined so that (14) is a second-order accurate approximation of (4) at $x_{J}$ and $x_{J+1}$. The analysis of local truncation error at $x_{J}$ and $x_{J+1}$ leads to a system of matrix equations whose solutions are $\Gamma$ 's, computed only one time during a preprocessing stage. At each time step, only some matrix-vector multiplications (14) need to be done at irregular points, what is very low-cost. The IIM can be coupled with other schemes: Zhang has demonstrated that numerical solutions are improved when a TVD scheme is used in conjunction with the second-order IIM (14). Using limiter functions reduces the order of accuracy but helps to dampen oscillations and to reduce phase errors. The IIM can be developed too to higher orders for coupling with high-orders schemes (like WENO 5).

However, the use of the IIM has some drawbacks. For identical material properties on both sides of $\alpha$, special formula (14) recover the original Lax-Wendroff scheme; even in the general case of distinct material properties, the second-order IIM is dispersive. So, if formula (14) are used at irregular points and coupled with a TVD scheme (that is not dispersive), numerical dispersion is introduced at two points per interface: for a high number of interfaces, it introduces a large amount of numerical artefacts. In a general manner, building an IIM that mimics the properties of the scheme $\mathcal{S}$ - for example ensuring TVD properties in the case of a TVD scheme, or minimizing oscillations in the case of the WENO 5 scheme - can become very complicated. Moreover, in the case of TVD scheme $(k=2)$, limiters introduce two additional irregular points, $x_{J-1}$ and $x_{J+2}$, where no interface method is used.

\section{The Explicit Jump Immersed Interface Method (EJIIM):}

A modification and extension of the IIM has been developed by Wiegmann and Bube [15] in the context of elliptic equations, called the Explicit Jump Immersed Interface Method (EJIIM). Corrections are added to the chosen scheme at irregular points, so that the local truncation error is maintained. In the limit case of identical material properties, corrections are equal to zero, recovering the original scheme. However, it is again difficult to calculate corrections in the case of a complicated scheme $\mathcal{S}$. Moreover, corrections are deduced from one-sided interpolations of $U$. When coupling the EJIIM and the Lax-Wendroff scheme, we have observed instabilities even for moderate contrasts. We suppose that they come from these non-symmetrical interpolations.

\section{The Explicit Simplified Interface Method (ESIM)}

\subsection{Introduction}

In the previous Section, we have stressed some properties, constraints and remarks about interface methods, summed up as follows:

- The order of accuracy of the scheme $\mathcal{S}$ must be maintained at all irregular points, whatever the position of the interface;

- We do not want to write explicitly a new scheme (like the IIM) or corrections depending on $\mathcal{S}$ (like the EJIIM);

- The scheme $\mathcal{S}$ must be recovered in the limit case of identical material properties on both sides of $\alpha$ (like the EJIIM); 
- Interpolations used by the interface method must be two-sided and symmetrical in relation to $\alpha$, in order to avoid instabilities.

So, we propose a new method called the Explicit Simplified Interface Method (ESIM). Its design wants to achieve the four previous remarks. For the rest of the Section, we will mainly consider a two-stage scheme $\mathcal{S}$.

\section{Modified solutions and modified values:}

The first part of the method consists in building, on each side of $\alpha$ and at time $t=$ $t_{n}$, a smooth extension $U^{*}\left(x, t_{n}\right)$ of the exact solution $U\left(x, t_{n}\right)$ on the other side. These extensions are called modified solutions. This is schematized in Figure 2 in the case of a

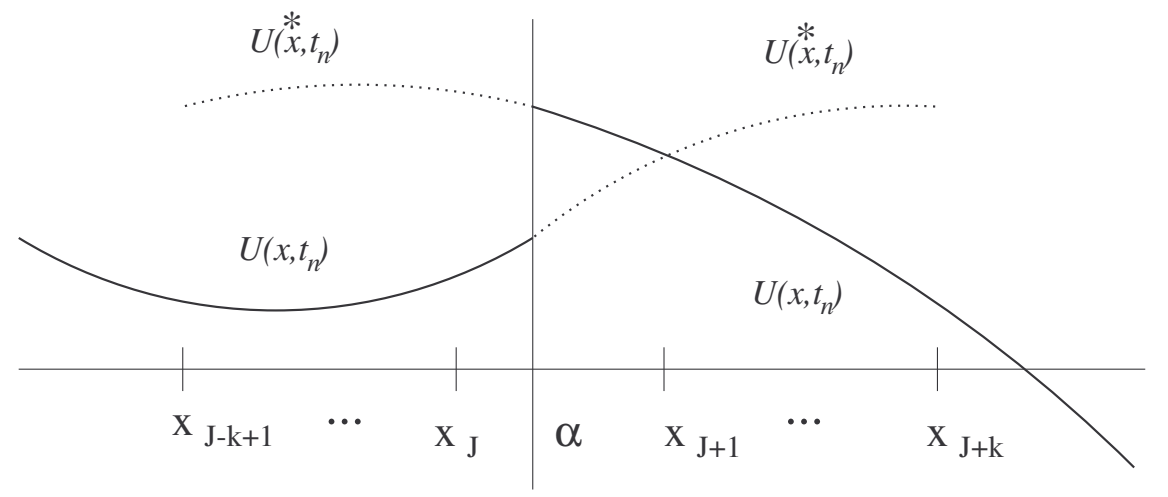

Figure 2: Exact solution $U\left(x, t_{n}\right)(-)$ and modified solutions $U^{*}\left(x, t_{n}\right)(\ldots)$.

scalar discontinuous fonction $U\left(x, t_{n}\right)$ (solid line): this is not the acoustic case ( $u$ and $p$ are continuous), but it is more clear. Modified solutions $U^{*}\left(x, t_{n}\right)$ are drawn in dotted lines and are defined so that functions:

$\tilde{U}_{1}\left(x, t_{n}\right)=\left\{\begin{array}{l}U\left(x, t_{n}\right) \text { if } x_{J-2 k+1} \leq x<\alpha \\ U^{*}\left(x, t_{n}\right) \text { if } \alpha \leq x \leq x_{J+k}\end{array} \quad \tilde{U}_{2}\left(x, t_{n}\right)=\left\{\begin{array}{l}U^{*}\left(x, t_{n}\right) \text { if } x_{J-k+1} \leq x \leq \alpha \\ U\left(x, t_{n}\right) \text { if } \alpha<x \leq x_{J+2 k}\end{array}\right.\right.$

are smooth up to an arbitrary order. Recall that $k$ is the width of the scheme $\mathcal{S}$ : so, $\tilde{U}_{1}\left(x, t_{n}\right)$ and $\tilde{U}_{2}\left(x, t_{n}\right)$ are defined on the range of values used for time-marching respectively at leftsided and at right-sided irregular points. Let us examine the first function $\tilde{U}_{1}\left(x, t_{n}\right)$ and the modified solution $U^{*}\left(x, t_{n}\right)$ on the right (15). We impose the following conditions:

$$
m=0, \ldots, 2 p-1, \quad \frac{\partial^{m}}{\partial x^{m}} U^{*}\left(\alpha, t_{n}\right)=\frac{\partial^{m}}{\partial x^{m}} U\left(\alpha^{-}, t_{n}\right),
$$

so that $\tilde{U}_{1}$ is in $\mathcal{C}^{2 p-1}$ on $\left[x_{J-2 k+1}, x_{J+k}\right]$. The integer $p$ is discussed further $(p$ has obviously nothing to do here with the acoustic pressure). The condition (16) is valid only for a sufficiently smooth initial value $U_{0}(x)=U(x, 0)$ : for $U_{0}(x)$ in $\mathcal{C}^{s}$, the exact solution $U(x, t)$ is in $\mathcal{C}^{s}$ on the left side $(x<\alpha)$ and on the right side $(x>\alpha)$. Limit values $\frac{\partial^{m}}{\partial x^{m}} U\left(\alpha^{-}, t_{n}\right)$ can be defined up to $m=s$, and (16) is well-defined under the condition $2 p-1 \leq s$. One way to ensure (16) is to write the modified solution on the right $U^{*}\left(x, t_{n}\right)$ as a polynomial:

$$
U^{*}\left(x, t_{n}\right)=\sum_{m=0}^{2 p-1} \frac{(x-\alpha)^{m}}{m !} \frac{\partial^{m}}{\partial x^{m}} U\left(\alpha^{-}, t_{n}\right)
$$


In fact, we can only obtain estimations of limit values $\frac{\partial^{m}}{\partial x^{m}} U\left(\alpha^{-}, t_{n}\right)$. Therefore, only estimations of $U^{*}\left(x, t_{n}\right)$ and $\tilde{U}_{1}\left(x, t_{n}\right)$, based on numerical values, can be found. Actually, these estimations will be avoided: modified values (i.e. values at right-sided irregular points of the estimation of $\left.U^{*}\left(x, t_{n}\right)\right)$ are determined directly and explicitly from numerical values. A similar discussion holds for $U^{*}\left(x, t_{n}\right)$ and modified values on the left, with limit values $\frac{\partial^{m}}{\partial x^{m}} U\left(\alpha^{+}, t_{n}\right)$.

\section{Using modified values:}

The second part of the method consists in using the scheme $\mathcal{S}$ everywhere, but some modified values are used for time-marching at irregular points. Suppose that $x_{i}$ is an irregular point. The key idea is to apply at $x_{i}$ the scheme $\mathcal{S}$ on values of the smooth function $\tilde{U}_{1}\left(x, t_{n}\right)$ if $x_{i} \leq \alpha$ (resp. $\tilde{U}_{2}\left(x, t_{n}\right)$ if $x_{i}>\alpha$ ). Thus the scheme $\mathcal{S}$ uses at $x_{i}$ numerical values at points on the same side of the interface as $x_{i}$ (as usually), and modified values at points on the other side of the interface. Remarks of the beginning of the Section have been taken into account in the following way:

- From a minimal number of numerical values $U_{l}^{n}$ 's used for the determination of modified values $U_{j}^{*}$ 's, the order of the couplage "scheme $\mathcal{S}+$ ESIM" at irregular points is the same as the order of the scheme $\mathcal{S}$ at regular points. See the Section 3-4 for the demonstration.

- Unlike the IIM or the EJIIM, the scheme $\mathcal{S}$ is modified implicitly. All the difficulty of the method is transferred on the determination of modified values (see Section 32 ), which is explicit and very easy (see (25) and (29)). Coupling WENO 5 with the ESIM is not harder than coupling Lax-Wendroff with the ESIM. It justifies the name "simplified".

- In the borderline case where material properties are the same on both sides of $\alpha$, $U_{i}^{n}=U_{i}^{*}$ at all irregular points (see Section 3-3). Then, the scheme $\mathcal{S}$ in homogeneous medium is completely recovered.

- Determinations of modified values are symmetrical in relation to $\alpha$. The stability is discussed qualitatively in Section 3-6.

\subsection{Construction of modified values}

\section{Construction of modified values on the right:}

Limit values $\frac{\partial^{m}}{\partial x^{m}} U\left(\alpha^{-}, t_{n}\right)$ required for $U^{*}\left(x, t_{n}\right)(17)$ are now estimated. Taylor series expansions of $2 p$-th order are written around $\alpha$ :

$$
i=J-p+1, \ldots, J+p, \quad U\left(x_{i}, t_{n}\right)=\sum_{m=0}^{2 p-1} \frac{\left(x_{i}-\alpha\right)^{m}}{m !} \frac{\partial^{m}}{\partial x^{m}} U\left(\alpha^{ \pm}, t_{n}\right)+O\left(\Delta x^{2 p}\right),
$$

where $\alpha^{ \pm}$refers to $\alpha^{-}$if $x<\alpha$ and to $\alpha^{+}$otherwise. The notation $O\left(\Delta x^{\lambda}\right)$, generally used for a scalar, refers here to a vector of an arbitrary size (depending on the context) whose entries are $O\left(\Delta x^{\lambda}\right)$ scalars. From jump conditions (11), we get:

$$
m=0, \ldots, 2 p-1, \quad \frac{\partial^{m}}{\partial x^{m}} U\left(\alpha^{+}, t_{n}\right)=D_{m} \frac{\partial^{m}}{\partial x^{m}} U\left(\alpha^{-}, t_{n}\right) .
$$

According to the position of $x_{i}$, equations (18) are:

$$
\begin{aligned}
& i=J-p+1, \ldots, J, \quad U\left(x_{i}, t_{n}\right)=\sum_{m=0}^{2 p-1} \frac{\left(x_{i}-\alpha\right)^{m}}{m !} \frac{\partial^{m}}{\partial x^{m}} U\left(\alpha^{-}, t_{n}\right)+O\left(\Delta x^{2 p}\right) \\
& i=J+1, \ldots, J+p, \quad U\left(x_{i}, t_{n}\right)=\sum_{m=0}^{2 p-1} \frac{\left(x_{i}-\alpha\right)^{m}}{m !} D_{m} \frac{\partial^{m}}{\partial x^{m}} U\left(\alpha^{-}, t_{n}\right)+O\left(\Delta x^{2 p}\right) .
\end{aligned}
$$


Equations (20) are written using a matrix formulation:

$$
\left(\begin{array}{c}
U\left(x_{J-p+1}, t_{n}\right) \\
\vdots \\
U\left(x_{J+p}, t_{n}\right)
\end{array}\right)=\mathcal{M}_{p, p}\left(\begin{array}{c}
U\left(\alpha^{-}, t_{n}\right) \\
\vdots \\
\frac{\partial^{2 p-1}}{\partial x^{2 p-1}} U\left(\alpha^{-}, t_{n}\right)
\end{array}\right)+O\left(\Delta x^{2 p}\right),
$$

where $\mathcal{M}_{p, q}$ is defined as a $(2 p)$-by- $(2 q)$ block matrix with 2 -by-2 blocks $(n=1, \ldots, 2 q)$ :

$$
\mathcal{M}_{p, q}[m, n]= \begin{cases}\frac{\left(x_{J-p+m}-\alpha\right)^{n-1}}{(n-1) !} I_{2} & \text { if } m \in[1, p] \\ \frac{\left(x_{J-p+m}-\alpha\right)^{n-1}}{(n-1) !} D_{n-1} & \text { if } m \in[p+1,2 p] .\end{cases}
$$

Even if $q=p$ in (21), the general definition of $\mathcal{M}_{p, q}$ (22) is useful in Section 3-6. Limit values $\frac{\partial^{m}}{\partial x^{m}} U\left(\alpha^{-}, t_{n}\right)$ could be obtained by inverting $(21)$, leading to $U^{*}\left(x, t_{n}\right)$ and $\tilde{U}_{1}\left(x, t_{n}\right)$. However, exact values and truncation errors are unknown. Exact values $U\left(x_{i}, t_{n}\right)$ are then replaced in (21) by numerical values $U_{i}^{n}$ and the vector of truncation errors is eliminated. Limit values $\frac{\partial^{m}}{\partial x^{m}} U\left(\alpha^{-}, t_{n}\right)$ are replaced by numerical estimations: for the sake of simplicity, these estimations are written $\frac{\partial^{m}}{\partial x^{m}} U_{1}^{-}$. The superscript 1 indicates that they are used for the construction of $\tilde{U}_{1}$, the operator $\frac{\partial^{m}}{\partial x^{m}}$ is only symbolical, and the superscript - refers to $\alpha^{-}$. Then we write:

$$
\left(\begin{array}{c}
U_{J-p+1}^{n} \\
\vdots \\
U_{J+p}^{n}
\end{array}\right)=\mathcal{M}_{p, p}\left(\begin{array}{c}
U_{1}^{-} \\
\vdots \\
\frac{\partial^{2 p-1}}{\partial x^{2 p-1}} U_{1}^{-}
\end{array}\right)
$$

Estimations of the limit values are obtained by inverting (23). It leads to an estimation of the modified solution (17) and to modified values:

$$
i=J+1, \ldots, J+k, \quad U_{i}^{*}=\sum_{m=0}^{2 p-1} \frac{\left(x_{i}-\alpha\right)^{m}}{m !} \frac{\partial^{m}}{\partial x^{m}} U_{1}^{-} .
$$

Using (23), we obtain explicitly $U_{i}^{*}$ 's on the right:

$$
i=J+1, \ldots, J+k, \quad U_{i}^{*}=\left(1, \ldots, \frac{\left(x_{i}-\alpha\right)^{2 p-1}}{(2 p-1) !}\right) \mathcal{M}_{p, p}^{-1}\left(\begin{array}{c}
U_{J-p+1}^{n} \\
\vdots \\
U_{J+p}^{n}
\end{array}\right) .
$$

\section{Construction of modified values on the left:}

The same method is used to define $\tilde{U}_{2}\left(x, t_{n}\right)$ and to compute modified values on the left. $\frac{\partial^{m}}{\partial x^{m}} U_{2}^{+}$refers to a numerical estimation of $\frac{\partial^{m}}{\partial x^{m}} U\left(\alpha^{+}, t_{n}\right)$. We write:

$$
\left(\begin{array}{c}
U_{J-p+1}^{n} \\
\vdots \\
U_{J+p}^{n}
\end{array}\right)=\mathcal{N}_{p, p}\left(\begin{array}{c}
U_{2}^{+} \\
\vdots \\
\frac{\partial^{2 p-1}}{\partial x^{2 p-1}} U_{2}^{+}
\end{array}\right)
$$

where $\mathcal{N}_{p, q}$ is a $(2 p)$-by- $(2 q)$ block matrix with 2 -by-2 blocks $(n=1, \ldots, 2 q)$ :

$$
\mathcal{N}_{p, q}[m, n]= \begin{cases}\frac{\left(x_{J-p+m}-\alpha\right)^{n-1}}{(n-1) !} D_{n-1}^{-1} & \text { if } m \in[1, p] \\ \frac{\left(x_{J-p+m}-\alpha\right)^{n-1}}{(n-1) !} I_{2} & \text { if } m \in[p+1,2 p] .\end{cases}
$$


Modified values on the left are:

$$
i=J-k+1, \ldots, J, \quad U_{i}^{*}=\sum_{m=0}^{2 p-1} \frac{\left(x_{i}-\alpha\right)^{m}}{m !} \frac{\partial^{m}}{\partial x^{m}} U_{2}^{+} .
$$

Using (26), $U_{i}^{*}$ 's on the left are obtained explicitly:

$$
i=J-k+1, \ldots, J, \quad U_{i}^{*}=\left(1, \ldots, \frac{\left(x_{i}-\alpha\right)^{2 p-1}}{(2 p-1) !}\right) \mathcal{N}_{p, p}^{-1}\left(\begin{array}{c}
U_{J-p+1}^{n} \\
\vdots \\
U_{J+p}^{n}
\end{array}\right)
$$

\subsection{Using modified values: ESIM $p-p$}

At an irregular point $x_{j}$, time-marching of a two-stage scheme $\mathcal{S}$ uses modified values at points on the other side of $\alpha$ as $x_{j}$. It is written abstractly (cf (13)):

$$
\begin{aligned}
& J-k+1 \leq j \leq J, \quad U_{j}^{n+1}=U_{j}^{n}+\mathcal{H}\left(U_{j-k}^{n}, \ldots, U_{J}^{n}, U_{J+1}^{*}, \ldots, U_{j+k}^{*}\right) \\
& J+1 \leq j \leq J+k, \quad U_{j}^{n+1}=U_{j}^{n}+\mathcal{H}\left(U_{j-k}^{*}, \ldots, U_{J}^{*}, U_{J+1}^{n}, \ldots, U_{j+k}^{n}\right) .
\end{aligned}
$$

This method is referred to as ESIM $p-p$. The application of ESIM to multi-stage schemes like WENO 5 is obvious. The construction of modified values at one stage uses numerical values at the previous stage. For the couplage "WENO $5+\operatorname{ESIM} p-p$ ", the procedure is repeated four times during a time step (i.e. at each Runge-Kutta integration).

Matrices $\mathcal{M}_{p, p}^{-1}$ and $\mathcal{N}_{p, p}^{-1}(25),(29)$ are computed explicitly only one time, during a preprocessing step. At each time step, only matrix-vector multiplications (25) and (29) need to be done. The computational cost is very low and is comparable to the cost of the IIM. We can show that $\mathcal{M}_{p, p}$ and $\mathcal{N}_{p, p}$ can be inverted - whatever the position of the interface and the values of physical parameters - by calculating their determinant. For example, let us see the case of $\mathcal{M}_{1,1}$. Setting:

$$
\epsilon=\frac{\alpha-J \Delta x}{\Delta x}
$$

we get:

$$
\operatorname{det} \mathcal{M}_{1,1}=\left[(1-\epsilon) \frac{\rho^{-}}{\rho^{+}}\left(\frac{c^{-}}{c^{+}}\right)^{2}+\epsilon\right]\left[(1-\epsilon) \frac{\rho^{+}}{\rho^{-}}+\epsilon\right] \Delta x^{2}
$$

which is always different from zero for positive values of $\rho^{ \pm}$and $c^{ \pm}$, and for $0 \leq \epsilon<1$. It has been verified too for $\mathcal{M}_{p, p}$ and $\mathcal{N}_{p, p}$ up to $p=3$.

For identical properties on both sides of $\alpha, D_{m}=I_{2}$ (10). For a right-sided irregular point $x_{i}$, we deduce from (22), (23) and (24):

$$
i=J+1, \ldots, J+k, \quad U_{i}^{*}=U_{i}^{n},
$$

if $k \leq p$. A similar property holds for left-sided irregular points. Therefore, the scheme $\mathcal{S}$ in homogeneous medium is completely recovered under the condition $k \leq p$.

\subsection{Local truncation error analysis}

Let $x_{j}$ be a left-sided irregular point $\left(x_{j} \leq \alpha\right)$. For a given two-stage scheme $\mathcal{S}$, we seek the local truncation error $\mathcal{L}_{1}\left(x_{j}, t_{n}\right)$ of the couplage "scheme $\mathcal{S}+$ ESIM $p-p$ " at $x_{j}$. Firstly, $\mathcal{L}_{1}\left(x_{j}, t_{n}\right)$ is developed. Secondly, an auxiliary problem is defined, whose solution and local truncation error $\mathcal{L}_{2}\left(x_{j}, t_{n}\right)$ are known. Comparing $\mathcal{L}_{2}\left(x_{j}, t_{n}\right)$ and $\mathcal{L}_{1}\left(x_{j}, t_{n}\right)$ leads to an explicit value of $\mathcal{L}_{1}\left(x_{j}, t_{n}\right)$. 


\section{Couplage "scheme $\mathcal{S}+$ ESIM $p-p$ ":}

Time-marching at $x_{j}$ is based on modified values (30), leading to:

$$
\underbrace{\frac{1}{\Delta t}\left(U_{j}^{n+1}-U_{j}^{n}\right)}_{\mathcal{L}_{1}^{1}}-\underbrace{\frac{1}{\Delta t} \mathcal{H}\left(U_{j-k}^{n}, \ldots, U_{J}^{n}, U_{J+1}^{*}, \ldots, U_{j+k}^{*}\right)}_{\mathcal{L}_{1}^{2}}=0 .
$$

The local truncation error $\mathcal{L}_{1}\left(x_{j}, t_{n}\right)$ is obtained by replacing by its exact value each numerical value that appears explicitly or implicitly in (34). The structure of (34) leads to write $\mathcal{L}_{1}\left(x_{j}, t_{n}\right)$ as the difference of two quantities $\mathcal{L}_{1}^{1}\left(x_{j}, t_{n}\right)$ and $\mathcal{L}_{1}^{2}\left(x_{j}, t_{n}\right)$. The first part $\mathcal{L}_{1}^{1}\left(x_{j}, t_{n}\right)$ is obtained from Taylor series expansions of $U\left(x_{j}, t_{n+1}\right)$ at $t_{n}$ :

$$
\begin{aligned}
\mathcal{L}_{1}^{1}\left(x_{j}, t_{n}\right) & =\frac{1}{\Delta t}\left[U\left(x_{j}, t_{n+1}\right)-U\left(x_{j}, t_{n}\right)\right] \\
& =\sum_{m=1}^{2 p-1} \frac{\Delta t^{m-1}}{m !} \frac{\partial^{m}}{\partial t^{m}} U\left(x_{j}, t_{n}\right)+O\left(\Delta x^{2 p-1}\right) .
\end{aligned}
$$

Recall that $\Delta t$ and $\Delta x$ are linked by the CFL number (12): $O\left(\Delta t^{\lambda}\right)=O\left(\Delta x^{\lambda}\right)$ for all $\lambda$. For the second part $\mathcal{L}_{1}^{2}\left(x_{j}, t_{n}\right)$ of $\mathcal{L}_{1}\left(x_{j}, t_{n}\right)$, Taylor series are written at $\alpha^{-}$on the left side $(i=j-k+1, \ldots, J)$ :

$$
U\left(x_{i}, t_{n}\right)=\sum_{m=0}^{2 p-1} \frac{\left(x_{i}-\alpha\right)^{m}}{m !} \frac{\partial^{m}}{\partial x^{m}} U\left(\alpha^{-}, t_{n}\right)+O\left(\Delta x^{2 p}\right) .
$$

On the right side $(i=J+1, \ldots, J+k)$, modified values $U_{i}^{*}$ 's are replaced by $\mathcal{U}^{*}\left(x_{i}, t_{n}\right)(25)$ :

$$
\mathcal{U}^{*}\left(x_{i}, t_{n}\right)=\left(1, \ldots, \frac{\left(x_{i}-\alpha\right)^{2 p-1}}{(2 p-1) !}\right) \mathcal{M}_{p, p}^{-1}\left(\begin{array}{c}
U\left(x_{J-p+1}, t_{n}\right) \\
\vdots \\
U\left(x_{J+p}, t_{n}\right)
\end{array}\right) .
$$

From (21), we get:

$$
\begin{aligned}
\mathcal{U}^{*}\left(x_{i}, t_{n}\right) & =\left(1, \ldots, \frac{\left(x_{i}-\alpha\right)^{2 p-1}}{(2 p-1) !}\right) \mathcal{M}_{p, p}^{-1}\left[\mathcal{M}_{p, p}\left(\begin{array}{c}
U\left(\alpha^{-}, t_{n}\right) \\
\vdots \\
\frac{\partial^{2 p-1}}{\partial x^{2 p-1}} U\left(\alpha^{-}, t_{n}\right)
\end{array}\right)+O\left(\Delta x^{2 p}\right)\right] \\
& =\sum_{m=0}^{2 p-1} \frac{\left(x_{i}-\alpha\right)^{m}}{m !} \frac{\partial^{m}}{\partial x^{m}} U\left(\alpha^{-}, t_{n}\right)+O\left(\Delta x^{2 p}\right) .
\end{aligned}
$$

The estimation of the error in (38) is based on two results. Firstly, we can determine the order of magnitude for the entries in the block vector:

$$
\tau=\mathcal{M}_{p, p}^{-1} O\left(\Delta x^{2 p}\right)={ }^{t}\left(O\left(\Delta x^{2 p}\right), \ldots, O(\Delta x)\right) .
$$

A similar result is shown in [2]. Secondly, we have obviously:

$$
\left(1, \ldots, \frac{\left(x_{i}-\alpha\right)^{2 p-1}}{(2 p-1) !}\right) \tau=O\left(\Delta x^{2 p}\right) .
$$

Transferring values (36) and (38) into $\mathcal{L}_{1}^{2}\left(x_{j}, t_{n}\right)$ leads to:

$$
\begin{array}{r}
\mathcal{L}_{1}^{2}\left(x_{j}, t_{n}\right)=\frac{1}{\Delta t} \mathcal{H}\left(\sum_{m=0}^{2 p-1} \frac{\left(x_{j-k}-\alpha\right)^{m}}{m !} \frac{\partial^{m}}{\partial x^{m}} U\left(\alpha^{-}, t_{n}\right)+O\left(\Delta x^{2 p}\right), \ldots,\right. \\
\left., \ldots, \sum_{m=0}^{2 p-1} \frac{\left(x_{j+k}-\alpha\right)^{m}}{m !} \frac{\partial^{m}}{\partial x^{m}} U\left(\alpha^{-}, t_{n}\right)+O\left(\Delta x^{2 p}\right)\right) .
\end{array}
$$


The discrete operator $\mathcal{H}$ is continuous [7], hence:

$$
\begin{aligned}
\mathcal{L}_{1}^{2}\left(x_{j}, t_{n}\right)= & \frac{1}{\Delta t} \mathcal{H}\left(\sum_{m=0}^{2 p-1} \frac{\left(x_{j-k}-\alpha\right)^{m}}{m !} \frac{\partial^{m}}{\partial x^{m}} U\left(\alpha^{-}, t_{n}\right), \ldots\right. \\
& \left., \ldots, \sum_{m=0}^{2 p-1} \frac{\left(x_{j+k}-\alpha\right)^{m}}{m !} \frac{\partial^{m}}{\partial x^{m}} U\left(\alpha^{-}, t_{n}\right)\right)+O\left(\Delta x^{2 p-1}\right) .
\end{aligned}
$$

We recall:

$$
\mathcal{L}_{1}\left(x_{j}, t_{n}\right)=\mathcal{L}_{1}^{1}\left(x_{j}, t_{n}\right)-\mathcal{L}_{1}^{2}\left(x_{j}, t_{n}\right)
$$

\section{Auxiliary problem:}

Let us consider the function:

$$
V(x, t)=\sum_{\lambda=0}^{2 p-1} \frac{(x-\alpha)^{\lambda}}{\lambda !} \frac{\partial^{\lambda}}{\partial x^{\lambda}} U\left(\alpha^{-}, t\right),
$$

where $U(x, t)$ is the solution of $U_{t}+A^{-} U_{x}=0$. We can easily verify that $V(x, t)$ is smooth across $\alpha$ and is the solution of the following Cauchy problem:

$$
\left\{\begin{array}{l}
V_{t}+A^{-} V_{x}=\frac{(x-\alpha)^{2 p-1}}{(2 p-1) !} \frac{\partial^{2 p}}{\partial x^{2 p}} U\left(\alpha^{-}, t\right) \\
V_{0}(x)=V(x, 0)=\sum_{\lambda=0}^{2 p-1} \frac{(x-\alpha)^{\lambda}}{\lambda !} \frac{\partial^{\lambda}}{\partial x^{\lambda}} U\left(\alpha^{-}, 0\right)
\end{array}\right.
$$

A condition on $U_{0}(x)$, slightly more strict than $2 p-1 \geq s$, is then required: limit values $\frac{\partial^{2 p}}{\partial x^{2 p}} U\left(\alpha^{ \pm}, t\right)$ must be defined for all $t$. The scheme $\mathcal{S}$ is used for the resolution of (45), so that a local truncation error $\mathcal{L}_{2}\left(x_{j}, t_{n}\right)$ is defined. For the exact solution (44) of (45), we can develop $\mathcal{L}_{2}\left(x_{j}, t_{n}\right)$ :

$$
\mathcal{L}_{2}\left(x_{j}, t_{n}\right)=\underbrace{\frac{V\left(x_{j}, t_{n+1}\right)-V\left(x_{j}, t_{n}\right)}{\Delta t}}_{\mathcal{L}_{2}^{1}\left(x_{j}, t_{n}\right)}-\underbrace{\frac{1}{\Delta t} \mathcal{H}\left(V\left(x_{j-k}, t_{n}\right), \ldots, V\left(x_{j+k}, t_{n}\right)\right)}_{\mathcal{L}_{2}^{2}\left(x_{j}, t_{n}\right)} .
$$

A Taylor expansion of $V\left(x_{j}, t_{n+1}\right)$ is written around $t_{n}$ :

$$
\mathcal{L}_{2}^{1}\left(x_{j}, t_{n}\right)=\sum_{m=1}^{2 p-1} \frac{\Delta t^{m-1}}{m !} \frac{\partial^{m}}{\partial t^{m}} V\left(x_{j}, t_{n}\right)+O\left(\Delta x^{2 p-1}\right) .
$$

For $m=1, \ldots, 2 p-1,(44)$ leads to:

$$
\begin{aligned}
\frac{\partial^{m}}{\partial t^{m}} V\left(x_{j}, t_{n}\right)= & \frac{\partial^{m}}{\partial t^{m}}\left(\sum_{\lambda=0}^{2 p-1-m} \frac{\left(x_{j}-\alpha\right)^{\lambda}}{\lambda !} \frac{\partial^{\lambda}}{\partial x^{\lambda}} U\left(\alpha^{-}, t_{n}\right)\right. \\
& \left.+\left(x_{j}-\alpha\right)^{2 p-m} \sum_{\lambda=2 p-m}^{2 p-1} \frac{\left(x_{j}-\alpha\right)^{\lambda-(2 p-m)}}{\lambda !} \frac{\partial^{\lambda}}{\partial x^{\lambda}} U\left(\alpha^{-}, t_{n}\right)\right) \\
= & \frac{\partial^{m}}{\partial t^{m}}\left(\sum_{\lambda=0}^{2 p-1-m} \frac{\left(x_{j}-\alpha\right)^{\lambda}}{\lambda !} \frac{\partial^{\lambda}}{\partial x^{\lambda}} U\left(\alpha^{-}, t_{n}\right)+O\left(\Delta x^{2 p-m}\right)\right)
\end{aligned}
$$

Hence, we have:

$$
\mathcal{L}_{2}^{1}\left(x_{j}, t_{n}\right)=\sum_{m=1}^{2 p-1} \frac{\Delta t^{m-1}}{m !} \frac{\partial^{m}}{\partial t^{m}}\left(\sum_{\lambda=0}^{2 p-1-m} \frac{\left(x_{j}-\alpha\right)^{\lambda}}{\lambda !} \frac{\partial^{\lambda}}{\partial x^{\lambda}} U\left(\alpha^{-}, t_{n}\right)\right)+O\left(\Delta x^{2 p-1}\right) .
$$


For each $m$, Taylor series expansions of $U\left(x_{j}, t_{n}\right)$ are written around $\alpha^{-}$:

$$
\sum_{\lambda=0}^{2 p-1-m} \frac{\left(x_{j}-\alpha\right)^{\lambda}}{\lambda !} \frac{\partial^{\lambda}}{\partial x^{\lambda}} U\left(\alpha^{-}, t_{n}\right)=U\left(x_{j}, t_{n}\right)+O\left(\Delta x^{2 p-m}\right),
$$

hence, we have:

$$
\mathcal{L}_{2}^{1}\left(x_{j}, t_{n}\right)=\sum_{m=1}^{2 p-1} \frac{\Delta t^{m-1}}{m !} \frac{\partial^{m}}{\partial t^{m}} U\left(x_{j}, t_{n}\right)+O\left(\Delta x^{2 p-1}\right) .
$$

For the computation of $\mathcal{L}_{2}^{2}\left(x_{j}, t_{n}\right), V\left(x_{j}, t_{n}\right)$ is replaced by its exact value (44):

$$
\mathcal{L}_{2}^{2}\left(x_{j}, t_{n}\right)=\frac{1}{\Delta t} \mathcal{H}\left(\sum_{m=0}^{2 p-1} \frac{\left(x_{j-k}-\alpha\right)^{m}}{m !} \frac{\partial^{m}}{\partial x^{m}} U\left(\alpha^{-}, t_{n}\right), \ldots, \sum_{m=0}^{2 p-1} \frac{\left(x_{j+k}-\alpha\right)^{m}}{m !} \frac{\partial^{m}}{\partial x^{m}} U\left(\alpha^{-}, t_{n}\right)\right) .
$$

We recall:

$$
\mathcal{L}_{2}\left(x_{j}, t_{n}\right)=\mathcal{L}_{2}^{1}\left(x_{j}, t_{n}\right)-\mathcal{L}_{2}^{2}\left(x_{j}, t_{n}\right)
$$

\section{Conclusion:}

From $(35),(42),(51)$ and $(52), \mathcal{L}_{1}\left(x, j, t_{n}\right)$ and $\mathcal{L}_{2}\left(x, j, t_{n}\right)$ are compared:

$$
\left.\begin{array}{l}
\mathcal{L}_{1}^{1}\left(x_{j}, t_{n}\right)=\mathcal{L}_{2}^{1}\left(x_{j}, t_{n}\right)+O\left(\Delta x^{2 p-1}\right) \\
\mathcal{L}_{1}^{2}\left(x_{j}, t_{n}\right)=\mathcal{L}_{2}^{2}\left(x_{j}, t_{n}\right)+O\left(\Delta x^{2 p-1}\right)
\end{array}\right\} \Rightarrow \mathcal{L}_{1}\left(x_{j}, t_{n}\right)=\mathcal{L}_{2}\left(x_{j}, t_{n}\right)+O\left(\Delta x^{2 p-1}\right) .
$$

$V(x, t)$ is smooth on $\left[x_{j-k}, x_{j+k}\right]$ at each time $t$. Moreover, we have supposed that the scheme $\mathcal{S}$ is $r$-th order accurate. So, we get $\mathcal{L}_{2}\left(x_{j}, t_{n}\right)=O\left(\Delta x^{r}\right)$ [10]. Thus, the couplage "scheme $\mathcal{S}+$ ESIM $p-p$ " is $r$-th order accurate under the following condition:

$$
2 p-1 \geq r .
$$

A similar result can be obtained for multistage schemes like WENO 5.

\subsection{Choice of $p$}

We seek the smallest values of $p$ that optimize the couplage "scheme $\mathcal{S}+$ ESIM $p-p$ ". The main constraints and results previously found are summed up as follows:

- $s \geq 2 p-1$ (required for the definition of $U^{*}\left(x, t_{n}\right)$ );

- $2 p-1 \geq r \Rightarrow \mathcal{L}_{1}=r$;

- $p \geq k \Rightarrow U_{i}^{*}=U_{i}^{n}$ if $\rho^{-}=\rho^{+}$and $c^{-}=c^{+}$;

Recall that $r$ and $k$ are respectively the order and the width of the scheme, $\mathcal{L}_{1}$ is the local truncation order of the couplage "scheme $\mathcal{S}+$ ESIM $p-p$ " at irregular points and $s$ is the smoothness of the initial value $U_{0}(x)=U(x, 0)$. It leads to:

$$
\begin{aligned}
& p=\max \left[k, r+1-E\left(\frac{r+1}{2}\right)\right] \\
& s=2 p-1,
\end{aligned}
$$

where $E(x)$ is the greatest integer less than or equal to $x$. For greater values of $p$, the precision does not increase, whereas the computational cost grows. As a consequence, LaxWendroff and TVD schemes are associated with ESIM 2-2, and WENO 5 is associated with ESIM 33. We recall that left values and right values of $\frac{\partial^{2 p}}{\partial x^{2 p}} U_{0}(x)$ must be defined everywhere. 


\subsection{Stability}

\section{Introduction:}

We do not propose a theory of stability for the couplage "scheme $\mathcal{S}+$ ESIM $p-p$ ". Nevertheless, we have considered this problem in detail through computations, for various positions of the interface, physical parameters and CFL numbers.

We have never observed instabilities in the case "WENO 5+ ESIM 33" up to CFL=1, even for very high contrasts (like water/air) and for an interface very close to a grid point, or on a grid point $(\epsilon=0)$. In each case, measures of errors have shown a fifth-order convergence of the couplage (see Section 4).

The couplages "Lax-Wendroff + ESIM 2-2" and "TVD + ESIM 2-2" are stable too except in two limit cases:

$$
\begin{aligned}
& \rho^{-} \gg \rho^{+}, c^{-} \gg c^{+} \text {and } \epsilon \rightarrow 1^{-} \\
& \rho^{-} \ll \rho^{+}, c^{-} \ll c^{+} \text {and } \epsilon \rightarrow 0^{+} .
\end{aligned}
$$

We have not obtained theoretical limit values of $\epsilon, \rho^{ \pm}$and $c^{ \pm}$, and it remains an interesting open question. However, we can express some remarks. Firstly, this problem of stability does exist too in the case of the IIM, with the same limit values of $\epsilon, \rho^{ \pm}$and $c^{ \pm}$. Secondly, the behaviour of Lax-Wendroff and TVD schemes coupled with ESIM 2-2 are the same: instabilities appear exactly from the same limit values of $\epsilon, \rho^{ \pm}$and $c^{ \pm}$. Thirdly, the value of $p$ has an influence on the stability. For example, coupling Lax-Wendroff scheme with ESIM $p-p(p=1,2,3)$ in the case water/air leads to instabilities for the following limit values of $\epsilon$ :

$$
\begin{array}{ll}
p=1: & \epsilon \geq 0.99 \\
p=2: & \epsilon \geq 0.999 \\
p=3: & \epsilon \geq 0.9999 .
\end{array}
$$

However, increasing $p$ is not a satisfactory solution: it is not well-matched to second-order schemes like Lax-Wendroff and TVD.

\section{Least-square resolution:}

To solve the previous problematic cases, we propose another determination of modified values that does not produce instabilities. The process of ESIM 2-2 - described in Section 3-2 - is shortly recalled: use of four numerical values on both sides of $\alpha$, estimation of spatial derivatives of $U$ up to the third-order. We propose now to estimate those four limit values with six numerical values of $U$ on both sides of $\alpha$. So, for the construction of modified values on the right, we write:

$$
\left(\begin{array}{c}
U_{J-2}^{n} \\
\vdots \\
U_{J+3}^{n}
\end{array}\right)=\mathcal{M}_{3,2}\left(\begin{array}{c}
U_{1}^{-} \\
\vdots \\
\frac{\partial^{3}}{\partial x^{3}} U_{1}^{-}
\end{array}\right)
$$

where $\mathcal{M}_{3,2}$ is the rectangular matrix (22) with $p=3$ and $q=2$. The equation (59) is solved by least-square, so that modified values on the right are:

$$
i=1,2, \quad U_{i}^{*}=\left(1, \ldots, \frac{\left(x_{i}-\alpha\right)^{3}}{3 !}\right)\left({ }^{t} \mathcal{M}_{3,2} \mathcal{M}_{3,2}\right)^{-1}{ }^{t} \mathcal{M}_{3,2}\left(\begin{array}{c}
U_{J-2}^{n} \\
\vdots \\
U_{J+3}^{n}
\end{array}\right) .
$$

The same procedure is applied for modified values on the left; this is logically called ESIM 3-2. Doing so, we have never observed instabilities, whatever the physical parameters and the position of the interface. A similar least-square procedure: "Lax-Wendroff + ESIM 2-1" has been used for $\epsilon \geq 0.99$ without producing instabilities (cf (58)). 
The analysis of truncation error exposed in Section 3-4 is always valid. Coupling "LaxWendroff + ESIM 3-2" and "TVD + ESIM 3-2" is second-order accurate as it is confirmed by measures of errors. Explaining the stability of ESIM 3-2 remains another interesting question. Furthermore, this least-squares resolution will be systematically used in $2 \mathrm{D}$ and $3 \mathrm{D}$ application of the ESIM, as it will be exposed in a future paper.

\section{Numerical results}

Three numerical tests are proposed. The first one concerns the propagation of an acoustic wave across a single interface with moderate contrasts. The analysis of Section 3-4 is confirmed by measures of errors. The second test extends the previous study to very large contrasts. The last example introduces a future study of the ESIM in 2D and 3D. A study of the computational cost is not provided here, because it is almost negligible in the one-dimensional case

\subsection{A single interface}

The simple acoustic problem of a single interface $(\alpha=96.3 \mathrm{~m})$ is considered, with a $300 \mathrm{~m}$ long fluid medium and physical parameters:

$$
(\rho(x), c(x))= \begin{cases}\rho_{0}=1000 \mathrm{~kg} / \mathrm{m}^{3}, c_{0}=1500 \mathrm{~m} / \mathrm{s} & \text { if } \quad x \leq \alpha \\ \rho_{1}=1200 \mathrm{~kg} / \mathrm{m}^{3}, c_{1}=2800 \mathrm{~m} / \mathrm{s} & \text { if } \quad x>\alpha .\end{cases}
$$

Numerical experiments are performed on 400 grid points, with $\mathrm{CFL}=0.8$ in medium 1 . They are initialized by a spatially-bounded right-going wave:

$$
U_{0}(x)=-f_{0}(\xi)\left(\begin{array}{c}
\frac{1}{c_{0}} \\
\rho_{0}
\end{array}\right) .
$$

The maximal smoothness is required for the couplage "WENO $5+$ ESIM 33": in this case, $s=5(56)$. So, we use a $\mathcal{C}^{5}$ spatially-bounded sinusoidal function $f_{0}(\xi)$ :

$$
f_{0}(\xi)=\left\{\begin{array}{l}
\sin \left(\omega_{c} \xi\right)-\frac{21}{32} \sin \left(2 \omega_{c} \xi\right)+\frac{63}{768} \sin \left(4 \omega_{c} \xi\right)-\frac{1}{512} \sin \left(8 \omega_{c} \xi\right) \quad \text { if } 0<\xi<\frac{1}{f_{c}} \\
0 \text { else, } \quad \text { with } \xi=t_{0}-\frac{x}{c} .
\end{array}\right.
$$

Left and right values of sixth-order's derivatives of $U_{0}(x)$ are defined everywhere (as needed in Section 3-4). The central frequency is $f_{c}=50 \mathrm{~Hz}$, and $t_{0}=51 \mathrm{~ms}$. The initial value of the acoustic pressure, called afterwards the solution, is shown in Figure 3-a. After reaching the interface, the acoustic wave is transmitted and reflected. Figures 3-b, 3-c and 3 -d show numerical (...) and exact values (solid line) of the solution at $t=90 \mathrm{~ms}$.

The Figure 3-b shows the solution computed with the couplage "Lax-Wendroff + ESIM 2-2". The dispersive behavior of the Lax-Wendroff scheme is clearly seen in spurious oscillations. The Figure 3-c shows the solution "TVD + ESIM 2-2". The use of a flux-limiter reduces oscillations but flattens the crests of the wave. The Figure 3 -d shows the solution "WENO 5 + ESIM 33". The acoustic wave is very well resolved.

The Table 4.1 shows the errors in both $L_{\infty}$ and $L_{1}$ norms obtained at $t=90 \mathrm{~ms}$ with these schemes, with and without the ESIM. Computations are performed in double precision. In the case of WENO 5 scheme, the time-step has been adjusted to $\Delta t \sim(\Delta x)^{5 / 4}$ so that the fourth-order Runge-Kutta in time is effectively fifth-order. When no interface method is used, homogeneized values $\rho_{i}$ and $c_{i}$ of $\rho$ and $c$ are used at grid points $x_{i}: \rho_{i}$ is the arithmetic 


\begin{tabular}{|c|c|c|c|c|c|}
\hline Method & $\mathrm{N}$ & $L_{\infty}$ error & $L_{\infty}$ order & $L_{1}$ error & $L_{1}$ order \\
\hline \multirow[t]{6}{*}{ Lax-Wendroff } & 200 & $2.38 \mathrm{e} 00$ & - & $8.78 \mathrm{e}+1$ & - \\
\hline & 400 & $8.81 \mathrm{e}-1$ & 1.43 & $2.71 \mathrm{e}+1$ & 1.70 \\
\hline & 800 & $2.15 \mathrm{e}-1$ & 2.03 & $7.22 \mathrm{e} 00$ & 1.90 \\
\hline & 1600 & $5.99 \mathrm{e}-2$ & 1.84 & $2.06 \mathrm{e} 00$ & 1.81 \\
\hline & 3200 & $3.13 \mathrm{e}-2$ & 0.93 & $9.90 \mathrm{e}-1$ & 1.06 \\
\hline & 6400 & $1.77 \mathrm{e}-2$ & 0.82 & $5.73 \mathrm{e}-1$ & 0.79 \\
\hline \multirow{6}{*}{$\begin{array}{c}\text { Lax-Wendroff } \\
+ \\
\text { ESIM } 2-2\end{array}$} & 200 & $2.82 \mathrm{e} 00$ & - & $9.80 \mathrm{e}+1$ & - \\
\hline & 400 & $1.14 \mathrm{e} 00$ & 1.31 & $3.20 \mathrm{e}+1$ & 1.61 \\
\hline & 800 & $3.30 \mathrm{e}-1$ & 1.78 & $8.49 \mathrm{e} 00$ & 1.91 \\
\hline & 1600 & $7.80 \mathrm{e}-2$ & 2.08 & $2.13 \mathrm{e} 00$ & 1.99 \\
\hline & 3200 & $1.93 \mathrm{e}-2$ & 2.01 & $5.33 \mathrm{e}-1$ & 2.00 \\
\hline & 6400 & $4.82 \mathrm{e}-3$ & 2.00 & $1.33 \mathrm{e}-1$ & 2.00 \\
\hline \multirow[t]{6}{*}{ TVD } & 200 & $1.55 \mathrm{e} 00$ & - & $3.86 \mathrm{e}+1$ & - \\
\hline & 400 & $6.03 \mathrm{e}-1$ & 1.36 & $1.41 \mathrm{e}+1$ & 1.45 \\
\hline & 800 & $2.91 \mathrm{e}-1$ & 1.05 & $9.47 \mathrm{e} 00$ & 0.57 \\
\hline & 1600 & $1.14 \mathrm{e}-1$ & 1.34 & $3.28 \mathrm{e} 00$ & 1.53 \\
\hline & 3200 & $3.48 \mathrm{e}-2$ & 1.72 & $1.26 \mathrm{e} 00$ & 1.37 \\
\hline & 6400 & $2.10 \mathrm{e}-2$ & 0.72 & $6.94 \mathrm{e}-1$ & 0.86 \\
\hline TVD & 200 & $1.61 \mathrm{e} 00$ & - & $3.42 \mathrm{e}+1$ & - \\
\hline+ & 400 & $6.32 \mathrm{e}-1$ & 1.35 & $9.94 \mathrm{e} 00$ & 1.78 \\
\hline \multirow[t]{4}{*}{ ESIM 2-2 } & 800 & $2.18 \mathrm{e}-1$ & 1.53 & $2.47 \mathrm{e} 00$ & 2.01 \\
\hline & 1600 & $7.35 \mathrm{e}-2$ & 1.57 & $6.38 \mathrm{e}-1$ & 1.95 \\
\hline & 3200 & $2.44 \mathrm{e}-2$ & 1.59 & $1.59 \mathrm{e}-1$ & 2.00 \\
\hline & 6400 & $8.19 \mathrm{e}-3$ & 1.57 & $4.05 \mathrm{e}-2$ & 1.97 \\
\hline \multirow[t]{6}{*}{ WENO 5} & 200 & $1.15 \mathrm{e} 00$ & - & $3.29 \mathrm{e}+1$ & - \\
\hline & 400 & $2.20 \mathrm{e}-1$ & 2.39 & $6.12 \mathrm{e} 00$ & 2.42 \\
\hline & 800 & $1.30 \mathrm{e}-1$ & 0.76 & $4.35 \mathrm{e} 00$ & 0.49 \\
\hline & 1600 & $3.36 \mathrm{e}-2$ & 1.95 & $1.05 \mathrm{e} 00$ & 2.04 \\
\hline & 3200 & $4.10 \mathrm{e}-2$ & -0.28 & $9.59 \mathrm{e}-1$ & 0.14 \\
\hline & 6400 & $1.15 \mathrm{e}-2$ & 1.83 & $1.89 \mathrm{e}-1$ & 2.34 \\
\hline \multirow{6}{*}{$\begin{array}{c}\text { WENO } 5 \\
+ \\
\text { ESIM } 33\end{array}$} & 200 & $7.74 \mathrm{e}-1$ & - & $2.02 \mathrm{e}+1$ & - \\
\hline & 400 & $6.13 \mathrm{e}-2$ & 3.65 & $1.44 \mathrm{e} 00$ & 3.80 \\
\hline & 800 & $2.96 \mathrm{e}-3$ & 4.37 & $8.12 \mathrm{e}-2$ & 4.15 \\
\hline & 1600 & $1.23 \mathrm{e}-4$ & 4.58 & $2.99 \mathrm{e}-3$ & 4.76 \\
\hline & 3200 & $4.50 \mathrm{e}-6$ & 4.77 & $1.03 \mathrm{e}-4$ & 4.85 \\
\hline & 6400 & $1.55 \mathrm{e}-7$ & 4.86 & $3.43 \mathrm{e}-6$ & 4.90 \\
\hline
\end{tabular}

Table 1: Errors and orders of accuracy in Example 4-1. 

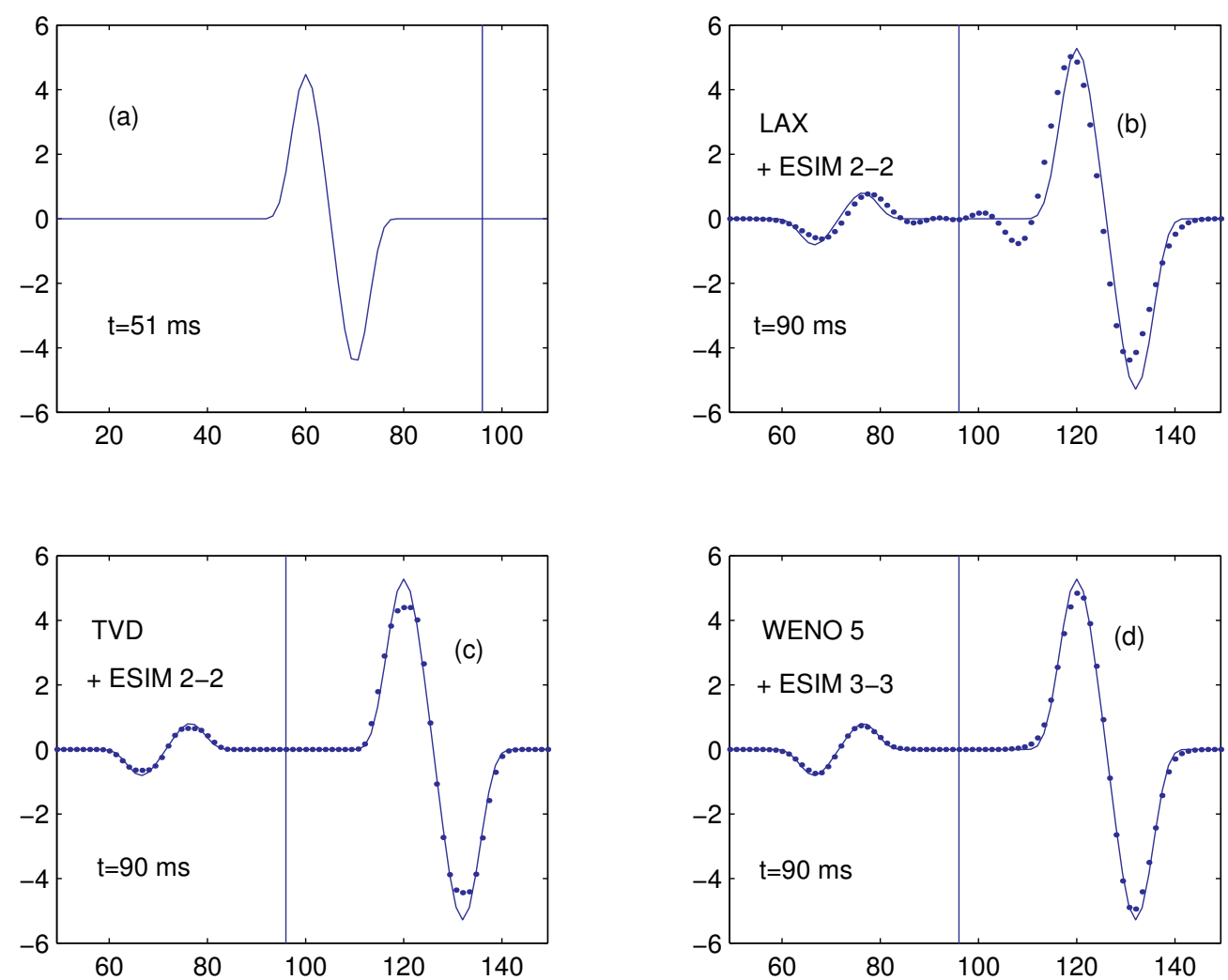

Figure 3: The exact solution at $t=51 \mathrm{~ms}(\mathrm{a})$; exact (solid line) and numerical solution $(\ldots)$ at $t=90$ ms: Lax-Wendroff + ESIM 2-2 (b), TVD + ESIM 2-2 (c), WENO $5+$ ESIM 3-3 (d).

average of $\rho(x)$ over $C_{i}=\left[x_{i-\frac{1}{2}}, x_{i+\frac{1}{2}}\right]$, and the bulk modulus $K_{i}$ is the harmonic average of $K(x)$ over $C_{i} ; c_{i}$ is deduced by $c_{i}=\sqrt{K_{i} / \rho_{i}}[6]$.

When the ESIM is not used, the order of accuracy is smaller than the theoretical order in homogeneous medium, and it changes a lot with the number of grid points. Using the ESIM (56) gives the order of accuracy in homogeneous medium:

- for Lax-Wendroff, 2 (in both norms),

- for TVD, 1.6 in $L_{\infty}$ norm and 2 in $L_{1}$ norm,

- for WENO 5, the orders tend to 5 (in both norms),

starting at about 400 grid points. Let us notice that the smoothness of $U_{0}(x)$ is fundamental to obtain the fifth-order accuracy of the couplage "WENO 5 + ESIM 33": only a 3.4 (in $L_{\infty}$ norm) and 2.4 (in $L_{1}$ norm) order is measured for a $\mathcal{C}^{2}$ function $f_{0}(\xi)$.

\subsection{Large contrast problem: interface water-air}

The previous study is extended to large contrast media. Material properties are:

$$
\begin{aligned}
& \rho_{0}=1000 \mathrm{~kg} / \mathrm{m}^{3}, \quad c_{0}=1450 \mathrm{~m} / \mathrm{s} \quad \text { (water) } \\
& \rho_{1}=1.3 \mathrm{~kg} / \mathrm{m}^{3}, \quad c_{1}=340 \mathrm{~m} / \mathrm{s} \quad \text { (air). }
\end{aligned}
$$



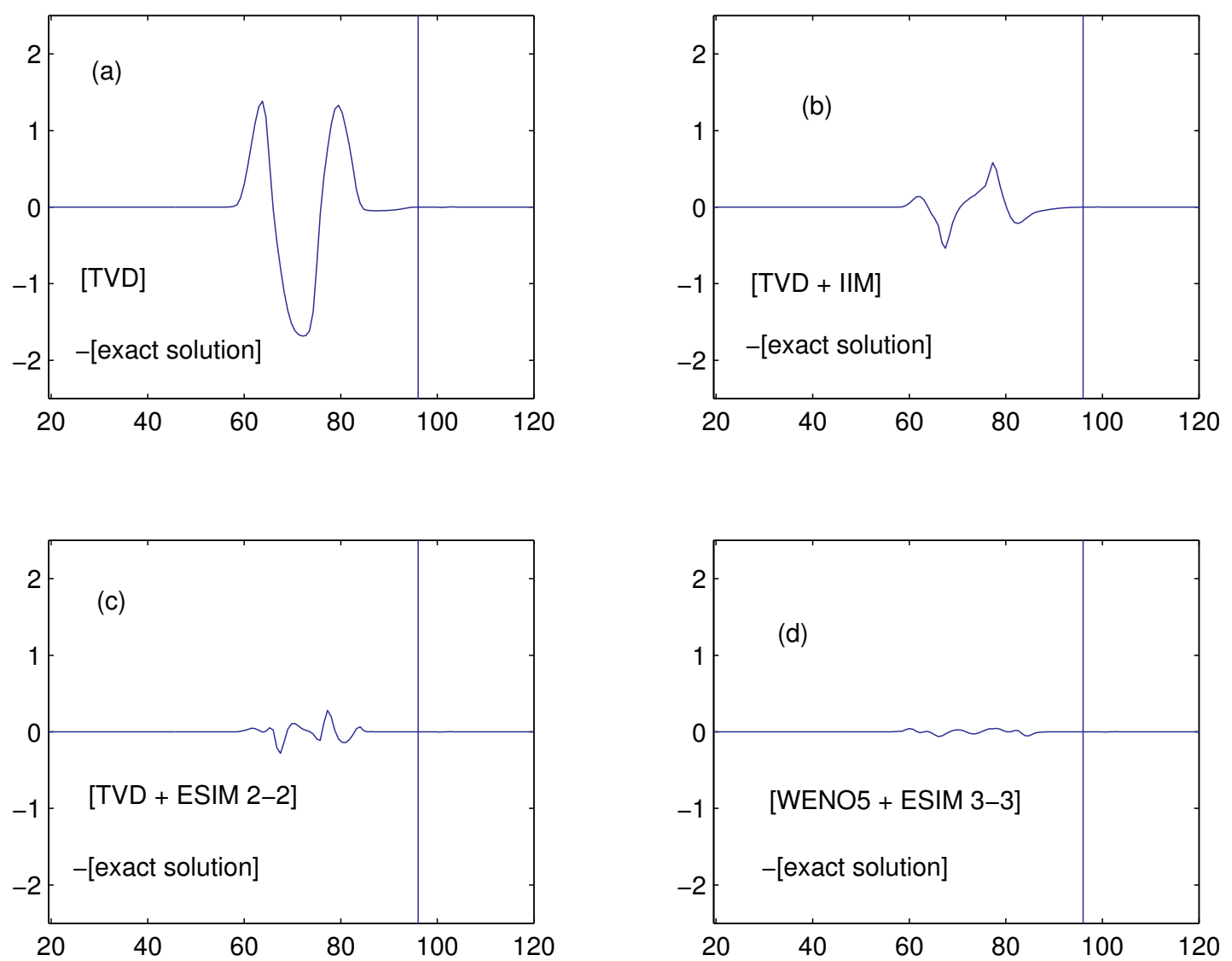

Figure 4: Differences between numerical and exact values of the solution at $t=90 \mathrm{~ms}$ (waterair): TVD scheme without an interface method (a), +IIM (b), +ESIM 2-2 (c), WENO $5+$ ESIM 33, from 20 up to $120 \mathrm{~m}$. The vertical line represents the position of the interface.
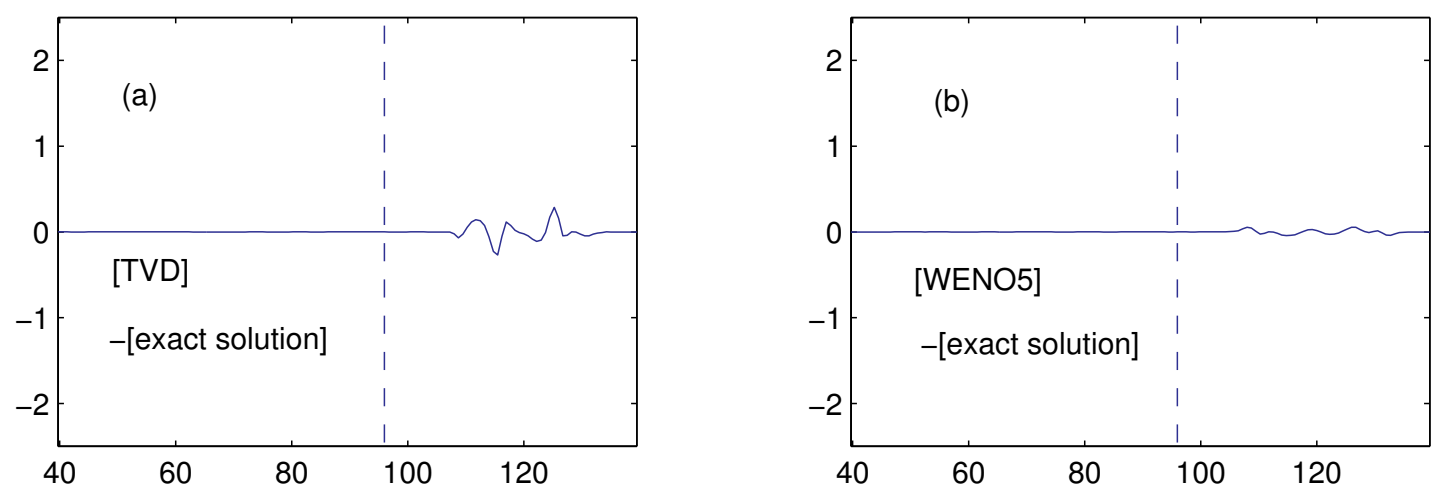

Figure 5: Differences between numerical and exact values of the solution at $t=90 \mathrm{~ms}$ (homogeneous water): TVD (a), WENO 5 (b), from 40 up to $140 \mathrm{~m}$. The dotted line recalls the position of the interface water-air in the example of Figure 4.

Differences between exact and numerical values of the solution computed by TVD and WENO 5 schemes with and without interface methods are plotted on Figure 4. Results are displayed from 20 up to $120 \mathrm{~m}$, at $t=90 \mathrm{~ms}$. The scale of the Y-axis has been almost 
amplified by a factor 3 (see Y-axis of Figure 3). Because of the impedance contrast, the wave is almost completely reflected: at this scale, the transmitted wave is not visible. Three subfigures show errors computed with a TVD scheme: without an interface method (a), with the IIM (b), with the ESIM 2-2 (c). Decreasing errors are observed and "WENO $5+$ ESIM 33 " is very accurate (Figure $4-\mathrm{d}$ ).

The Figure 5 shows errors computed with TVD and WENO 5 schemes in homogeneous water $\left(\rho_{0}, c_{0}\right)$ at $t=90 \mathrm{~ms}$ (obviously, no interface method is used). At this time, acoustic waves have traveled in water the same distance as reflected acoustic waves of Figure 4 . Dotted lines in Figure 5 recall the position of the interface in Figure 4.

It is instructive to compare Figures 4-c, 4-d respectively with Figures 5-a, 5-b, after performing symmetries of these last ones in relation to the $\mathrm{X}$-axis and to the dotted vertical line (these symmetries are due to the impedance contrast: $\rho_{1} c_{1}>\rho_{0} c_{0}$ and to the change in direction). Shapes and levels of errors are then very close, as it is theoretically. The presence of the interface does not introduce noticeable artefacts when the ESIM is used.

Measures of errors and orders of accuracy have been performed too for various positions of $\alpha$ and values of $\epsilon$ (31), confirming the analysis of Section 3-4. If Lax-Wendroff or TVD scheme is used, and for an interface very close to a grid point $(\epsilon>0.999)$, ESIM 3-2 is used to avoid instabilities (see Section 3-6).

\subsection{D plane wave}

As a last example, we provide a two-dimensional result. The key idea of the ESIM - determination and use of modified values at irregular points - remains obviously the same as in the one-dimensional case. The derivation of jump conditions, the construction of modified values by a least-square resolution and the analysis of the resulting scheme will be omitted here; the comparaison with other interface methods and the investigation of various cases (interface waves, elastic waves...) will be developed too in a future publication. Our goal here is only to show the improvement of the computation when the ESIM is used.

We use an unsplit high-resolution scheme with flux-limiters, called wave-propagation algorithm, developed by Langseth and LeVeque $[9,11]$. The acoustic case in a 2D heterogeneous fluid medium is studied in [9].
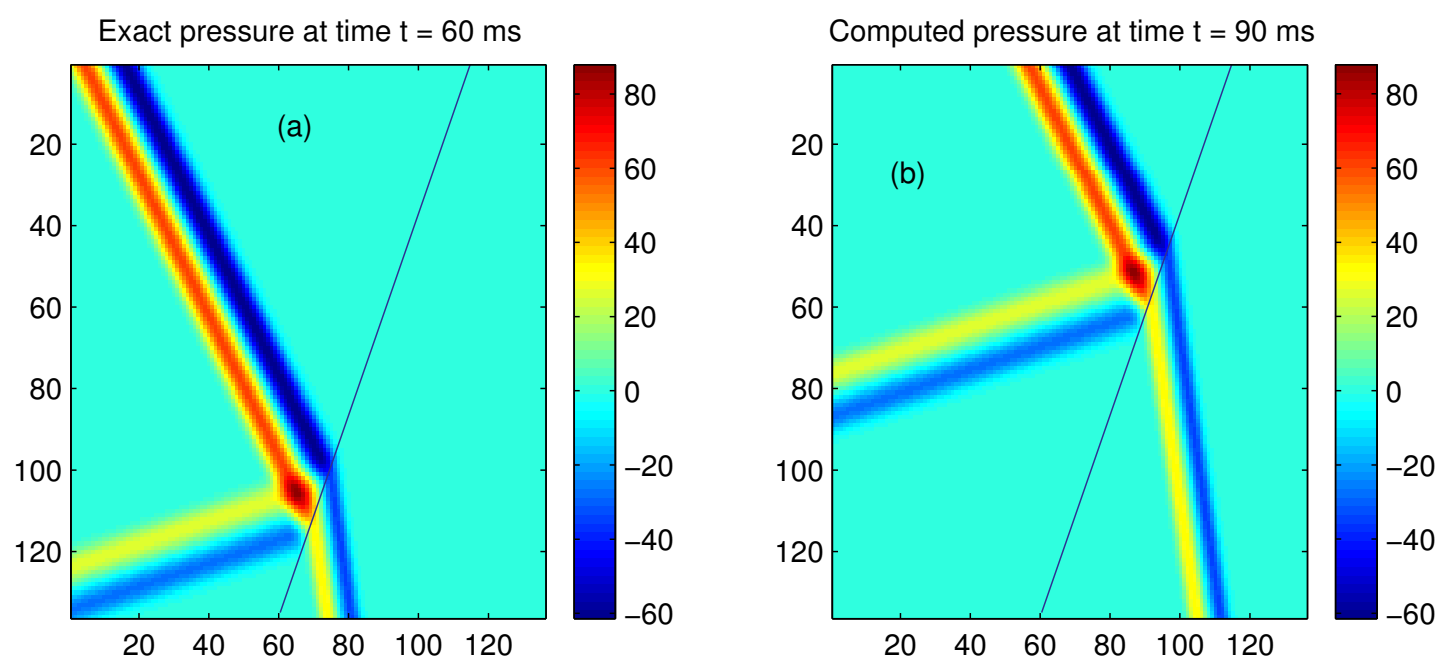

Figure 6: Plane wave hitting a plane interface that is not aligned with the grid. Exact values of the acoustic pressure at $t=60 \mathrm{~ms}$ (a) and computed values of the acoustic pressure at $t$ $=90 \mathrm{~ms}(\mathrm{~b})$. 

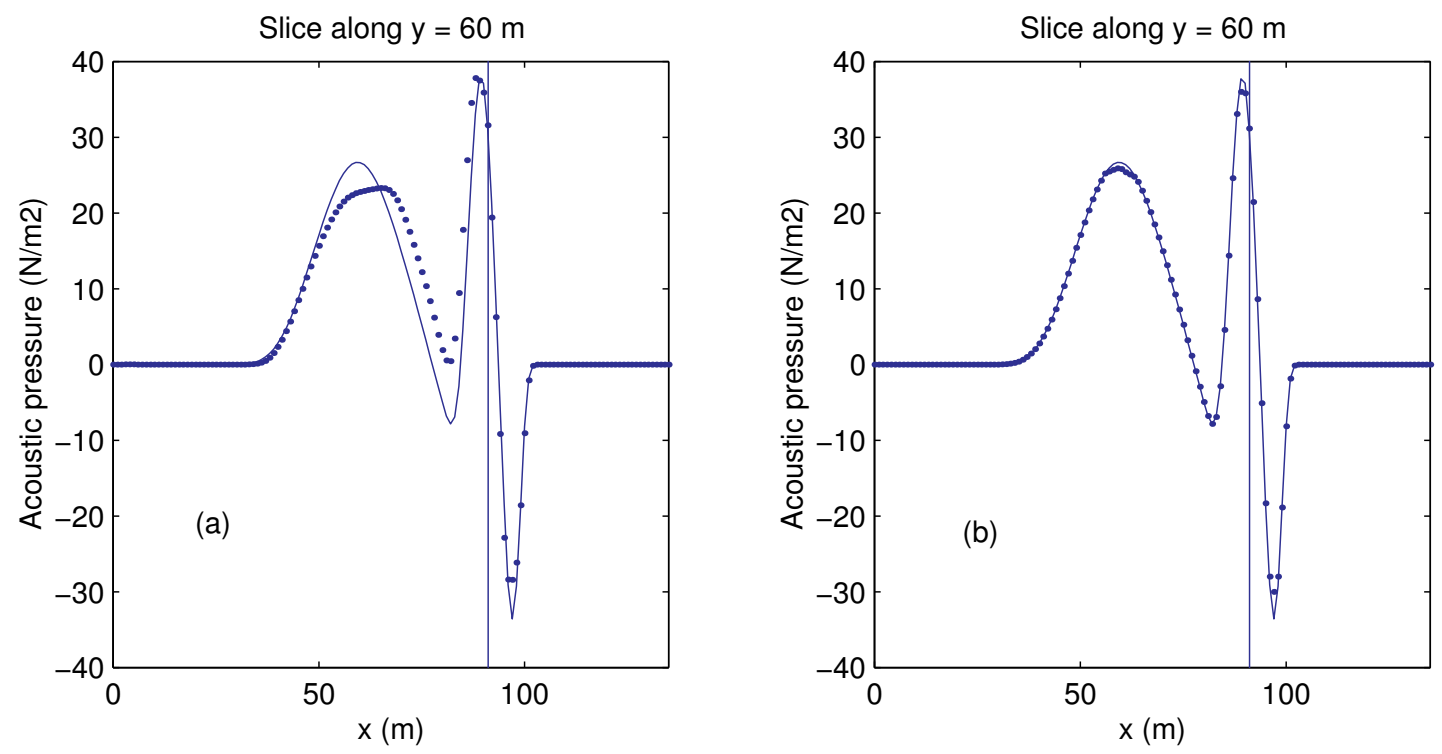

Figure 7: Plane wave hitting a plane interface that is not aligned with the grid. Values of the acoustic pressure computed by a Wave Propagation Algorithm without an interface method (a) and with the ESIM (b) at $t=90 \mathrm{~ms}$, along $\mathrm{y}=60 \mathrm{~m}$. Exact (solid line) and numerical solution $(\ldots)$.

We consider the case of a plane wave stricking a plane interface that is not aligned with the grid. Exact values of the acoustic pressure (called again the solution) of reflected and transmitted waves are easy to determine [1]. The interface is described by:

$$
\Gamma=\left\{M(x, y) / y=f(x)=\left(x-x_{0}\right) \tan \theta_{0}\right\}
$$

with $\theta_{0}=68$ degrees and $x_{0}=60.4 \mathrm{~m}$. Physical parameters are:

$$
(\rho(x, y), c(x, y))=\left\{\begin{array}{l}
\rho_{0}=1000 \mathrm{~kg} / \mathrm{m}^{3}, c_{0}=1500 \mathrm{~m} / \mathrm{s} \quad \text { if } \quad y>f(x) \\
\rho_{1}=800 \mathrm{~kg} / \mathrm{m}^{3}, c_{1}=1000 \mathrm{~m} / \mathrm{s} \quad \text { if } \quad y \leq f(x) .
\end{array}\right.
$$

The incident wave is based again on $f_{0}(\xi)$, with

$$
\xi=t_{0}-\frac{1}{c_{0}}\left(x \cos \theta_{i}+y \sin \theta_{i}\right)
$$

$t_{0}=60 \mathrm{~ms}, \theta_{i}=30$ degrees and $f_{c}=50 \mathrm{~Hz}$. Computations are performed on $136 \times 136$ grid points for a $135 \mathrm{~m} \times 135 \mathrm{~m}$ domain, with $\mathrm{CFL}=0.8 \mathrm{in}$ medium 0 (the wave propagation algorithm is stable up to CFL $=1$ in $2 \mathrm{D}$ ). Because of the stencil, two "ghost cells" lines are defined on each boundary of the domain. Exact values of the acoustic pressure and of acoustic velocities are assigned at ghostcells at each time step. See [9] for additional remarks.

Figure 6 shows initial values of the solution at $t=60 \mathrm{~ms}$, and the computed solution at $t=90 \mathrm{~ms}$. Figure 7 shows slices of the exact (solid line) and computed (...) reflected and transmitted waves along $y=60 \mathrm{~m}$ at $t=90 \mathrm{~ms}$, without the ESIM (a) and using the ESIM (b). In the first case, we take into account the interface by averaging the physical parameters to obtain values in each grid cell. Then, the solution is smeared across the interface. One can find a discussion about this problem in the case of Maxwell equations in [3]. Using an interface method like the ESIM avoids these numerical artefacts (Figure 7-b). 


\section{Conclusion}

A new interface method has been developed and presented in the 1D case, the Explicit Simplified Interface Method (ESIM). Modifying explicitly numerical values used by a numerical scheme at irregular points amounts to modify implicitly the scheme, so that its order is maintained at irregular points. The implementation of the ESIM can be divided into three independant parts:

- Writing of jump conditions. These ones only depend on the physical problem under study and on geometrical features of the interface;

- Construction of modified values during a preprocessing step. This algorithm depends on the scheme's width and order but not on its expression;

- Computation and use of modified values at each time step.

Qualities of the method have been verified through various numerical examples. The last example (Section 4-4) introduces a future discussion about 2D and 3D applications that we have developed. Interface methods on uniform cartesian grids like the ESIM are of general interest, not only for computational acoustics, but also for a wide class of problems like computational electromagnetics and fluid mechanics.

Acknowledgments: We thank Jean-Pierre Sessarego (LMA, Marseille) for his confidence and Paul Cristini for introducing us to interface methods. We are grateful to Philippe Angot and Murielle Guichaoua (IRPHEE, Marseille) for numerous suggestions concerning numerical schemes and mathematical background. We thank Arnaud de Ponnat for helpful and very interesting discussions about our method.

\section{References}

[1] L. M. Brekhovskikh and O. A. Godin, Acoustics of Layered Media 1- Plane and Quasi-Plane Waves, Springer-Verlag, Berlin Heidelberg, 1990.

[2] D. Calhoun, A Cartesian Grid Method for Solving the Streamfunction Vorticity Equations in Irregular Geometries, PhD. thesis, University of Washington, Seattle, WA, 1999.

[3] F. Collino And P. Joly and F. Millot, Fictitious domain method for unsteady problems: Application to electromagnetic scattering, J. Comput. Phys., 138 (1997), pp. 907-938.

[4] R. P. Fedkiw, B. Merriman, R. Donat, and S. Osher, The penultimate scheme for systems of conservation laws: finite-difference ENO with Marquina's flux splitting, UCLA CAM Report 96-18 (1996).

[5] D. Calhoun, High-Resolution Finite Volume Methods for Acoustics in a RapidlyVarying Heterogeneous Medium, Master's thesis, University of Washington, Seattle, WA, 1997.

[6] T.R. Fogarty and R.J. LeVeque, High-Resolution Finite Volume Methods for Acoustic Waves in Periodic and random Media, J. Acoust. Soc. Am., 106-1 (1999), pp. $17-28$.

[7] E. Godlewski and P. A. Raviart, Numerical Approximation of Hyperbolic Systems of Conservation Laws, Springer-Verlag, 1996.

[8] G. S. Jiang And C. W. Shu, Efficient implementation of weighted ENO schemes, J. Comput. Phys., 126 (1996), pp. 202-228. 
[9] J. O. LAngseth And R. J. LeVEque, A wave-propagation method for threedimensional hyperbolic conservation laws, J. Comput. Phys, 165 (2000), pp. 126-166.

[10] R. J. LeVeque, Numerical Methods for Conservation Laws, Birkhauser, 1990.

[11] R. J. LEVEQUE, Wave propagation algorithms for multi-dimensionnal hyperbolic systems, J. Comput. Phys., 13 (1997), pp. 327-353.

[12] Z. Li, The Immersed Interface Method - A Numerical Approach for Partial Differential Equations with Interfaces, PhD. thesis, University of Washington, Seattle, WA, 1994.

[13] Z. Li AND R. J. LeVEQue, The Immersed Interface Method for elliptic equations with discontinuous coefficients and singular sources, SIAM J. Num. Anal., 31 (1994), pp. 1019-1044.

[14] S. I. Rokhlin AND Y. J. WAng, Analysis of boundary conditions for elastic wave interaction with an interface between two solids, J. Acoust. Soc. Am., 89-2 (1991), pp. 503-515.

[15] A. Wiegmann, The Explicit Jump Immersed Interface Method and Interface Problems for Differential Equations, PhD. thesis, University of Washington, Seattle, WA, 1998.

[16] C. Zhang, Immersed Interface Method for Hyperbolic Systems of Partial Differential Equations with Discontinuous Coefficients, PhD. thesis, University of Washington, Seattle, WA, 1996.

[17] C. Zhang and R. J. LeVeque, The Immersed Interface Method for acoustic wave equations with discontinuous coefficients, Wave Motion, 25 (1997), pp. 237-263. 